\title{
On the $T^{2}$ control chart with estimated parameters
}

\author{
Jiaqi Chen ${ }^{1 *}$ and Hualong Yang ${ }^{2}$, Jianfeng $\mathrm{Yao}^{2}$ \\ ${ }^{1}$ Department of Mathematics, Harbin Institute of Technology \\ ${ }^{2}$ Department of Statistics and Actuarial Science, University of Hong Kong
}

November 20, 2016

\begin{abstract}
Statistical monitoring of multivariate processes is becoming increasingly important in modern manufacturing environments. Typical equipment may have multiple key variables to be measured continuously. Hotelling's $T^{2}$ chart was originally applied for monitoring the mean vector of multivariate quality measurements. In practical problems, estimated parameters are needed and their use will modify the properties of control charts. The Average Run Length (ARL), an indicator of the performance of the control charts, will be larger when the estimated parameters are used. As one contribution of the paper, we provide a rigorous proof of this phenomenon which has been reported in several empirical studies. Furthermore, in order to design an efficient $T^{2}$ chart with estimated parameters, it is necessary to have a method to calculate or approximate the ARL function. An existing approach in the literature is based on extensive Monte-Carlo simulations. In this paper, we propose a novel approach by providing an analytic approximation of the ARL function in the however limited case of univariate observations.
\end{abstract}

Keywords: Average Run Length; Estimated parameters; Multivariate statistical process control; $T^{2}$ chart

\footnotetext{
${ }^{*}$ Corresponding author. E-mail:chenjq1016@hit.edu.cn
} 


\section{Introduction}

Statistical monitoring of multivariate processes is becoming increasingly important in modern manufacturing environments. Typical equipment may have multiple key variables to be measured continuously. Hotelling's $T^{2}$ chart (Hotelling , 1947) was originally applied to the problem of monitoring the mean vector of multivariate quality measurements. In practice, parameters of the observation process need to be estimated first during a Phase I analysis. As a consequence, the use of such estimated parameters will modify the properties of control charts in Phase II study. The performance of multivariate charts with estimated parameters has been studied in the literature. Ryan (2011) analyzed the dependence of $T^{2}$ chart on estimated parameters. Lowry and Montgomery (1995) provided recommended sample sizes for multivariate $T^{2}$ control charts. A more detailed study on the effects of the parameter estimation on multivariate $T^{2}$ charts with $\chi^{2}$ based control limits was done by Nedumaran and Pignatiello (1999). Champ et al. (2005) studied the $T^{2}$ charts with corrected control limits. Jensen et al. (2006) and Bersimis et al. (2006) give a thorough review of the literature concerning the effects of estimation on univariate and multivariate charts with estimated parameters.

Throughout the paper, we assume that the base vector $\mathbf{X}$ of quality measurements is distributed as the $p$-variate normal distribution $\mathcal{N}_{p}\left(\mu, \Sigma_{0}\right)$. The process is said in-control when $\mu=\mu_{0}$, a preassigned mean vector. When the in-control parameters $\mu_{0}$ and $\Sigma_{0}$ are known, Hotelling's $T^{2}$ chart uses the statistics at time $k=1,2, \ldots$,

$$
T_{k}^{2}=n\left(\overline{\mathbf{X}}_{k}-\mu_{0}\right)^{\mathrm{T}} \Sigma_{0}^{-1}\left(\overline{\mathbf{X}}_{k}-\mu_{0}\right)
$$

where $\overline{\mathbf{X}}_{k}$ is the mean of the $k$-th sample with sample size $n$. The chart gives an out-ofcontrol signal at time $i$ when $T_{i}$ is above a control limit $h$.

A widely used method to measure the performance of a control chart is through the average run length $(A R L)$, which is the expected number of the plotted chart statistics before a signal is observed. When $\mu_{0}$ and $\Sigma_{0}$ are unknown, we replace them by some sample estimates for them. Sample estimators $\hat{\mu}_{0}$ and $\hat{\Sigma}_{0}$ are based on data acquired prior to the on-going inspection process. Assume that there are $m$ such independent random samples $\left\{\mathbf{X}_{i, 1}, \mathbf{X}_{i, 2}, \ldots, \mathbf{X}_{i, n}\right\}$ of size $n(i=1,2, \ldots, m)$ from an in-control process. Then the sample estimators $\hat{\mu}_{0}$ and $\hat{\Sigma}_{0}$ are:

$$
\begin{aligned}
& \hat{\mu}_{0}=\overline{\overline{\mathbf{X}}}=\frac{1}{m n} \sum_{i=1}^{m} \sum_{j=1}^{n} \mathbf{X}_{i, j} \\
& \hat{\Sigma}_{0}=\overline{\mathbf{S}}=\frac{1}{m(n-1)} \sum_{i=1}^{m} \sum_{j=1}^{n}\left(\mathbf{X}_{i, j}-\overline{\mathbf{X}}_{i}\right)\left(\mathbf{X}_{i, j}-\overline{\mathbf{X}}_{i}\right)^{\mathrm{T}}
\end{aligned}
$$


The $T^{2}$ statistics are then replaced with

$$
T_{k}^{2}=n\left(\overline{\mathbf{X}}_{k}-\hat{\mu}_{0}\right)^{\mathrm{T}} \hat{\Sigma}_{0}^{-1}\left(\overline{\mathbf{X}}_{k}-\hat{\mu}_{0}\right) .
$$

The whole procedure of $T^{2}$ chart is then divided into two phases. In Phase One, the chart is used to determine whether the $m$ independent random samples each with size $n$ is generated from an in-control process and then the parameter estimators can be obtained through these samples. In Phase Two, these estimated parameters are directly used in testing whether new sample points deviate from the in-control status based on some control limits. Sullivan and Woodall (1996) and Kim et al. (2003) have discussed the problem of adapting control charts for the preliminary analysis of multivariate observations and also recommend a method for preliminary analysis of multivariate observations that does not require any simulation for the control limit for Phase One. For Phase Two, the main task is to determine a control limit $h$ which keeps the in-control $A R L$ at a given level (e.g. 200 or 500). The out-of-control $A R L$ are also to be estimated and they should be kept as small as possible. If $\mu_{0}$ and $\Sigma_{0}$ are known, each statistic $T_{k}^{2}$ in $(1.1)(k=1,2, \ldots)$ follow a $\chi^{2}$ distribution independently with $p$ degree of freedom and the $R L$ of a chart is a geometric random variable. For example, the multivariate Shewart control chart has an upper control limit $h=\chi_{p, 1-\alpha}^{2}$ with an in-control $A R L=1 / \alpha$. Several authors provided an analysis on $\left\{T_{k}^{2}\right\}$ when sample estimators $\hat{\mu}_{0}$ and $\hat{\Sigma}_{0}$ are used as in (1.2). For example, Wierda (1994) showed that $[(m n-m-p+1) / p(m+1)(n-1)] T_{k}^{2}$ is distributed as a Fisher distribution $F_{p, m n-m-p+1}$. In Phase Two, if the subsequent statistics $T_{k}^{2}$ were independent at different time point $k=1,2, \ldots$, the control limit $h^{*}$ corresponding to an in-control $A R L=1 / \alpha$ would be

$$
h^{*}=\frac{p(m+1)(n-1)}{m(n-1)+1-p} F_{1-\alpha, p, m n-m-p+1,}, \quad \text { and } \quad \mathrm{P}\left(T_{1}^{2}>h^{*}\right)=\alpha,
$$

where $F_{1-\alpha, p, m n-m-p+1}$ is the $\alpha$-th upper quantile of $F_{p, m n-m-p+1}$. However, as estimators are used in place of known parameters, the $T_{k}^{2}$ 's become dependent so that with the control limit $h^{*}$ specified above, the actual in-control $A R L$ indeed differs from the target value $\alpha^{-1}$.

Champ et al. (2005) took the dependence among the $T_{k}^{2}$ into consideration and offered corrected control limit $h$ using intensive simulations. More precisely, using the sample estimators $\hat{\mu}_{0}$ and $\hat{\Sigma}_{0}$, the current statistic $T_{k}^{2}$ of (1.2) with inspected sample mean $\overline{\mathbf{X}}^{k}$ computed from the sample $\mathbf{X}^{k}=\left\{\mathbf{X}_{k, 1}, \mathbf{X}_{k, 2}, \ldots, \mathbf{X}_{k, n}\right\}$ can be written as:

$$
\begin{aligned}
& T_{k}^{2}=m(n-1)\left(\mathbf{Z}_{k}+\sqrt{n} \delta-\frac{1}{\sqrt{m}} \mathbf{Z}_{0}\right)^{\mathrm{T}} \mathbf{W}_{0}^{-1}\left(\mathbf{Z}_{k}+\sqrt{n} \delta-\frac{1}{\sqrt{m}} \mathbf{Z}_{0}\right) \\
& \mathbf{Z}_{0}=\sqrt{m n} \mathbf{P}_{0}^{-1}\left(\overline{\overline{\mathbf{X}}}-\mu_{0}\right) \sim \mathrm{N}_{p}(\mathbf{0}, \mathrm{I}), \mathbf{Z}_{k}=\sqrt{n} \mathbf{P}_{0}^{-1}\left(\overline{\mathbf{X}^{k}}-\mu_{0}\right) \sim \mathrm{N}_{p}(\mathbf{0}, \mathrm{I}), \delta=\mathbf{P}_{0}^{-1}\left(\mu-\mu_{0}\right), \\
& \mathbf{W}_{0}=m(n-1) \mathbf{P}_{0}^{-1} \overline{\mathbf{S}}\left(\mathbf{P}_{0}^{-1}\right)^{\mathrm{T}} \sim \operatorname{Wishart}_{p}(\mathrm{I}, m(n-1)) \text {, and the matrix } \mathbf{P}_{0} \text { is defined by } \\
& \text { the factorization } \Sigma_{0}=\mathbf{P}_{0} \mathbf{P}_{0}^{\mathrm{T}} \text {. Moreover, } \mathbf{Z}_{0}, \mathbf{Z}_{k} \text {, and } \mathbf{W}_{0} \text { are independent in this repre- }
\end{aligned}
$$


sentation. If we further use an orthogonal transformation $\mathbf{B}$ such that $\mathbf{B} \delta=(d, 0, \ldots, 0)^{\mathrm{T}}$, we have

$$
T_{k}^{2}=m(n-1)\left(\mathbf{Z}_{k}^{*}+\sqrt{n} d \mathbf{e}-\frac{1}{\sqrt{m}} \mathbf{Z}_{0}^{*}\right)^{\mathrm{T}} \mathbf{W}_{0}^{*-1}\left(\mathbf{Z}_{k}^{*}+\sqrt{n} d \mathbf{e}-\frac{1}{\sqrt{m}} \mathbf{Z}_{0}^{*}\right)
$$

where $\mathbf{e}$ is the $\mathrm{p} \times 1$ vector $(1,0, \ldots, 0)^{\mathrm{T}}, \mathbf{Z}_{0}^{*}=\mathbf{B Z}_{0} \sim \mathrm{N}_{p}(\mathbf{0}, \mathrm{I}), \mathbf{Z}_{k}^{*}=\mathbf{B Z}_{k} \sim \mathrm{N}_{p}(\mathbf{0}, \mathrm{I})$, $\mathbf{W}_{0}^{*}=\mathbf{B W}_{0} \mathbf{B}^{\mathrm{T}} \sim \mathrm{Wishart}_{p}(\mathrm{I}, m n-m)$ and they are independent. Notice that $d^{2}=$ $\|\delta\|^{2}=\left(\mu-\mu_{0}\right)^{\mathrm{T}} \Sigma_{0}^{-1}\left(\mu-\mu_{0}\right)$ is the non-central parameter. Two important features of the $T^{2}$ chart with estimated parameters emerge from this representation. First, the distribution of each $T_{k}^{2}$ depends on the unknown parameters $\mu_{0}$ and $\Sigma_{0}$ through $d$ only; second, even though the samples, say $k=1,2 \ldots$, in Phase II are independent, the statistics $\left\{T_{k}^{2}\right\}_{k \geqslant 1}$ are dependent since they share the same random variables $\mathbf{Z}_{0}^{*},\left\{\mathbf{Z}_{k}^{*}\right\}_{k \geqslant 1}$ and $\mathbf{W}_{0}^{*}$ which are functions of the estimated parameters. However, the $\left\{T_{k}^{2}\right\}_{k \geqslant 1}$ still have the same marginal distribution.

Despite the interesting representation (1.5), the joint distribution of $\left\{T_{k}^{2}\right\}_{k \geqslant 1}$ is far from being known. This makes it a particular challenge to determine a control limit $h$ for a given in-control $A R L$. One attempt consists of keeping the same control limit $h$ as if the parameters were known and not estimated, namely the traditional limit $h^{*}$. However, it has been reported in the literature that this seemingly natural approach inflates the actual $A R L_{0}$ (in-control) which becomes larger than the target $A R L_{0}$, see e.g. Bersimis et al. (2006). Moreover, Champ et al. (2005) provided detailed simulations to verify this phenomenon. As first main contribution of the paper, we will provide a formal proof of the phenomenon.

Furthermore, the design of an efficient $T^{2}$ chart with estimated parameters requires an appropriate method to calculate or approximate the $A R L$ function. Champ et al. (2005) proposes an effective approach where the $A R L$ function is tabulated using extensive Monte-Carlo simulation. Typically, given a target $A R L_{0}$, say 200 with $m$ samples of block length $n$ in Phase I, the corrected UCL $\bar{h}$ can be found by large size simulations and binary adjustments of $h$. Tables in Champ et al. (2005) report the corrected UCL $\bar{h}$ for $A R L_{0}=200$ and a wide range of parameters: dimension $2 \leqslant p \leqslant 10$, sample size $30 \leqslant m \leqslant 100$ and block length $3 \leqslant n \leqslant 15$.

Although this Monte-Carlo approach is valuable and precious, one may search for other approximation methods for several reasons. First, since the random $A R L$ function has a quite large variance, such simulation procedures may suffer from numerical instability. Second, as for all tabulating approaches, a practitioner may face a problem where the parameters $p, m$ and $n$ are not documented in the established tables. A method based on analytic approximation relaxes the restriction on $m$ and $n$ and provides a more convenient alternative to designing a $T^{2}$ chart. As second main contribution of this paper, we establish such an analytic approximation for the $A R L$ function. Due to the complexity of the problem, we limit ourselves to the univariate case $p=1$ and we establish a numerical method for the determination of a control limit $h$ when an arbitrary in-control $A R L$ is given. 


\section{Comparison between target and actual values of $A R L$ when using the traditional control limit}

For a given upper control limit $h$, the $A R L$ function of the $T^{2}$ chart is

$$
\begin{aligned}
A R L(h) & =\sum_{k=0}^{\infty} k \mathrm{P}(\text { Run length }=k)=\sum_{k=0}^{\infty} \mathrm{P}(\text { Run length }>k) \\
& =\sum_{k=0}^{\infty} \mathrm{P}\left(T_{1}^{2} \leqslant h, T_{2}^{2} \leqslant h, \ldots, T_{k-1}^{2} \leqslant h, T_{k}^{2} \leqslant h\right) .
\end{aligned}
$$

Meanwhile, the traditional control limit $h^{*}$ is derived by assuming the independence between the $T_{k}^{2}$ 's, which is

$$
A R L_{0} \simeq \sum_{k=0}^{\infty}\left\{\mathrm{P}\left(T_{1}^{2} \leqslant h^{*}\right)\right\}^{k}=\frac{1}{\mathrm{P}\left(T_{1}^{2}>h^{*}\right)}=\frac{1}{\alpha}
$$

where $h^{*}$ equals to the $\alpha$-th upper quantile of $T_{1}^{2}$ as given in (1.3). For example, if the target $A R L_{0}$ is 200 , one finds $\alpha=\frac{1}{200}$ and the value of $h^{*}$ is derived.

However, the statistics $T_{k}^{2}$ 's are indeed dependent. There will be therefore a bias between the actual $A R L$ and the target $A R L$ using the independence approximation (2.2) above. Actually, it has been observed for years that using estimated parameters leads to an in-control $A R L$ that is higher than the expected one.

To the best of our knowledge, this paper is the first to give a formal proof of this phenomenon.

Theorem 1. For the $T^{2}$ chart in (1.5) using estimated parameters $\hat{\mu}_{0}$ and $\hat{\Sigma}_{0}$, we have $A R L(h) \geqslant \frac{1}{P\left(T_{1}^{2}>h\right)}$ for all $h>0$. In particular, if the traditional control limit $h^{*}$ in (1.3) is used, then the actual in-control $A R L$ is always larger than the target $A R L$. i.e. $A R L_{0} \geqslant \frac{1}{\alpha}$.

Proof. By (2.1),

$$
A R L(h)=\sum_{k=0}^{\infty} \mathrm{P}\left(T_{1}^{2} \leqslant h, \ldots, T_{k}^{2} \leqslant h\right)=\sum_{k=0}^{\infty} \mathrm{E}\left\{\mathbf{1}_{\left(T_{1}^{2} \leqslant h\right)}, \ldots, \mathbf{1}_{\left(T_{k}^{2} \leqslant h\right)}\right\} .
$$

Here $\mathbf{1}_{A}$ denotes the indicator function of an event $A$. Note that in the representation (1.5), the $\left\{\mathbf{Z}_{k}^{*}\right\}_{k \geqslant 1}$ are independent of $\mathbf{Z}_{0}^{*}$ and $\mathbf{W}_{0}^{*}$. As a result, conditionally to $\mathbf{Z}_{0}^{*}$ and $\mathbf{W}_{0}^{*}$, the statistics $\mathbf{T}_{k}^{2}$ s are independent. Therefore, by the conditioning method,

$$
\begin{aligned}
\mathrm{E}\left\{\mathbf{1}_{\left(T_{1}^{2} \leqslant h\right)}, \ldots, \mathbf{1}_{\left(T_{k}^{2} \leqslant h\right)}\right\} & =\mathrm{E}\left\{\mathrm{E}\left(\mathbf{1}_{\left(T_{1}^{2} \leqslant h\right)}, \ldots, \mathbf{1}_{\left(T_{k}^{2} \leqslant h\right)} \mid \mathbf{Z}_{0}^{*}, \mathbf{W}_{0}^{*}\right)\right\} \\
& =\mathrm{E}\left[\left\{\mathrm{E}\left(\mathbf{1}_{\left(T_{1}^{2} \leqslant h\right)} \mid \mathbf{Z}_{0}^{*}, \mathbf{W}_{0}^{*}\right)\right\}^{k}\right] .
\end{aligned}
$$


After applying the Jensen's inequality $\mathrm{E}\left[X^{k}\right] \geqslant\{\mathrm{E}[X]\}^{k}$ to the nonnegative variables $X=\mathrm{E}\left(\mathbf{1}_{\left(T_{1}^{2} \leqslant h\right)} \mid \mathbf{Z}_{0}^{*}, \mathbf{W}_{0}^{*}\right)$ and observing that $\mathrm{E}[X]=\mathrm{E}\left[\mathbf{1}_{\left(T_{1}^{2} \leqslant h\right)}\right]=\mathrm{P}\left(T_{1}^{2} \leqslant h\right)$, we have

$$
\mathrm{E}\left\{\mathbf{1}_{\left(T_{1}^{2} \leqslant h\right)}, \ldots, \mathbf{1}_{\left(T_{k}^{2} \leqslant h\right)}\right\} \geqslant\left\{\mathrm{E}\left[\mathrm{E}\left(\mathbf{1}_{\left(T_{1}^{2} \leqslant h\right)} \mid \mathbf{Z}_{0}^{*}, \mathbf{W}_{0}^{*}\right)\right]\right\}^{k}=\left\{\mathrm{P}\left(T_{1}^{2} \leqslant h\right)\right\}^{k}
$$

Hence,

$$
A R L(h) \geqslant \sum_{k=0}^{\infty}\left\{\mathrm{P}\left(T_{1}^{2} \leqslant h\right)\right\}^{k}=\frac{1}{\mathrm{P}\left(T_{1}^{2}>h\right)} .
$$

In particular, for the in-control $A R L$ using traditional control limit as UCL,

$$
A R L\left(h^{*}\right) \geqslant \frac{1}{\mathrm{P}_{0}\left(T_{1}^{2}>h^{*}\right)}=\frac{1}{\alpha} .
$$

Theorem 1 formally establishes the fact reported in the literature (Bersimis et al. , 2006) that using the traditional limit $h^{*}$ in the presence of estimated parameters will inflates the in-control ARL. Moreover, we observe that Equation (2.5) shows that the $\operatorname{ARL}(h)$ function is increasing in $h$, so that the needed UCL $\bar{h}$ for a given target in-control ARL must be smaller than the traditional limit $h^{*}$. As for the out-of-control ARL, again by the monotonicity of the ARL function, the wrong use of $h^{*}$ instead of the needed UCL $\bar{h}$ will also increases the underlying out-of-control ARL.

\section{Analytic approximations for the in-control $A R L$ in the univariate case}

As explained in the introduction, we develop an approximation method for the in-control $A R L$ function with univariate observations. To begin with, we give an exact analytic expression of the in-control $A R L$.

Proposition 1. When $p=1$,

$$
A R L_{0}=E\left(\frac{1}{\bar{\Phi}\left(b_{0} \sqrt{h}+a_{0}\right)+\bar{\Phi}\left(b_{0} \sqrt{h}-a_{0}\right)}\right)
$$

where $a_{0}=\mathbf{Z}_{0}^{*} / \sqrt{m}, b_{0}=\sqrt{\mathbf{W}_{0}^{*}} / \sqrt{m(n-1)}, \bar{\Phi}(x)=\int_{x}^{\infty} \frac{1}{\sqrt{2 \pi}} \exp \left(-\frac{t^{2}}{2}\right) d t$ and $\mathbf{Z}_{0}^{*}, \mathbf{W}_{0}^{*}$ are the variables defined in (1.5).

Proof. First, we rewrite (1.5) when $p=1$ and assume all Phase II observation $\left\{T_{k}^{2}\right\}_{k=1,2, \ldots}$ are in-control $(d=0)$ : 


$$
T_{k}^{2}=\frac{m(n-1)}{\mathbf{W}_{0}^{*}}\left(\mathbf{Z}_{k}^{*}-\frac{1}{\sqrt{m}} \mathbf{Z}_{0}^{*}\right)^{2}=\left(\frac{\mathbf{Z}_{k}^{*}-a_{0}}{b_{0}}\right)^{2},
$$

where $a_{0}=\mathbf{Z}_{0}^{*} / \sqrt{m}, b_{0}=\sqrt{\mathbf{W}_{0}^{*}} / \sqrt{m(n-1)}$. Here $\mathbf{Z}_{0}^{*} \sim \mathrm{N}(\mathbf{0}, 1), \mathbf{W}_{0}^{*} \sim \chi_{m(n-1)}^{2}$. Particularly, $T_{1}^{2}=\left(\frac{\mathbf{z}_{1}^{*}-a_{0}}{b_{0}}\right)^{2}$.

Denote the conditional probability $\mathrm{P}^{*}=\mathrm{P}\left(T_{1}^{2}>h \mid \mathbf{Z}_{0}^{*}, \mathbf{W}_{0}^{*}\right) \in(0,1)$. Then based on the distribution of $T_{1}^{2}$ above, we have

$$
\begin{aligned}
\mathrm{P}^{*} & =\mathrm{P}\left(T_{1}<-\sqrt{h} \mid \mathbf{Z}_{0}^{*}, \mathbf{W}_{0}^{*}\right)+\mathrm{P}\left(T_{1}>\sqrt{h} \mid \mathbf{Z}_{0}^{*}, \mathbf{W}_{0}^{*}\right) \\
& =\mathrm{P}\left(\mathbf{Z}_{1}^{*}<-b_{0} \sqrt{h}+a_{0} \mid \mathbf{Z}_{0}^{*}, \mathbf{W}_{0}^{*}\right)+\mathrm{P}\left(\mathbf{Z}_{k}^{*}>b_{0} \sqrt{h}+a_{0} \mid \mathbf{Z}_{0}^{*}, \mathbf{W}_{0}^{*}\right) \\
& =\bar{\Phi}\left(b_{0} \sqrt{h}+a_{0}\right)+\bar{\Phi}\left(b_{0} \sqrt{h}-a_{0}\right) .
\end{aligned}
$$

According to (2.3), (2.4) and (3.3) above,

$$
\begin{aligned}
A R L_{0}(h) & =\sum_{k=0}^{\infty} \mathrm{E}\left[\left\{\mathrm{E}\left(\mathbf{1}_{\left(T_{1}^{2} \leqslant h\right)} \mid \mathbf{Z}_{0}^{*}, \mathbf{W}_{0}^{*}\right)\right\}\right]^{k} \\
& =\sum_{k=0}^{\infty} \mathrm{E}\left[\left\{\mathrm{P}\left(T_{1}^{2} \leqslant h \mid \mathbf{Z}_{0}^{*}, \mathbf{W}_{0}^{*}\right)\right\}\right]^{k} \\
& =\sum_{k=0}^{\infty} \mathrm{E}\left[1-\mathrm{P}^{*}\right]^{k}=\mathrm{E}\left(\frac{1}{\mathrm{P}^{*}}\right) \\
& =\mathrm{E}\left(\frac{1}{\bar{\Phi}\left(b_{0} \sqrt{h}+a_{0}\right)+\bar{\Phi}\left(b_{0} \sqrt{h}-a_{0}\right)}\right)
\end{aligned}
$$

We have obtained an analytic expression of $A R L_{0}$, which depends on the conditional probabilities $G\left(a_{0}, b_{0}, h\right)=\left(\bar{\Phi}\left(b_{0} \sqrt{h}+a_{0}\right)+\bar{\Phi}\left(b_{0} \sqrt{h}-a_{0}\right)\right)^{-1}$. We seek for an approximation of $G\left(a_{0}, b_{0}, h\right)$ in order to find an effective approximation of the $A R L$ function (3.1).

There is a traditional approximation to evaluate the complementary cumulative distribution function $\bar{\Phi}(x)$ (see Abramowitz and Stegun (1965)): 


$$
\begin{aligned}
\bar{\Phi}(x) & \simeq \frac{Z(x)}{x}\left\{1-\frac{1}{x^{2}}+\frac{1 \cdot 3}{x^{4}}-\cdots+\frac{(-1)^{N_{1}} \cdot 1 \cdot 3 \cdots\left(2 N_{1}-1\right)}{x^{2 N_{1}}}\right\} \\
& =Z(x) \sum_{i=0}^{N_{1}} \frac{(-1)^{i} \cdot 1 \cdot 3 \cdots(2 i-1)}{x^{2 i+1}}, \quad x \neq 0,
\end{aligned}
$$

where $Z(x)=(2 \pi)^{-1 / 2} e^{-x^{2} / 2}$ is the standard normal density function and $N_{1} \geq 1$ determines the degree of the expansion (here for $i=0$, the product $1 \cdot 3 \cdots(2 i-1)$ is empty so that its value equals to 1 by convention). The main idea is then to approximate the denominator of the $G$ function with (3.4).

Let $y=b_{0} \sqrt{h}=\sqrt{\frac{\mathbf{W}_{\mathbf{0}}^{*} h}{m(n-1)}}$ and $z=a_{0}=\frac{\mathbf{Z}_{0}^{*}}{\sqrt{m}}$ where $\mathrm{Z}_{0}^{*} \sim \mathrm{N}(\mathbf{0}, 1), \mathrm{W}_{0}^{*} \sim \chi_{m(n-1)}^{2}$. We find that $z$ is small with high probability since $\mathrm{E}(z)=0$ and $\operatorname{var}(z)=\frac{1}{m}$, where $m$ is usually large. Expanding $\bar{\Phi}$ around $y$, we can rewrite $G$ as

$$
G\left(a_{0}, b_{0}, h\right)=\frac{1}{2 \bar{\Phi}(y)+m(z, y)}=\frac{1}{2 \bar{\Phi}(y)}-l(z, y)
$$

where $m(z, y)$ and $l(z, y)$ are the errors associated to these approximations which are small compared with $\frac{1}{2 \bar{\Phi}(y)}$. Therefore, the procedure of approximating $G\left(a_{0}, b_{0}, h\right)$ can be decomposed into two steps. First, we estimate the main part $\frac{1}{2 \bar{\Phi}(y)}$. Second, we find an estimation for $l(z, y)$, which will helpfully lead to an approximation of $G$ and finally of the $A R L$ function in (3.1).

\subsection{Approximation of $\frac{1}{2 \Phi(y)}$}

We rewrite (3.4) as

$$
\bar{\Phi}(y) \backsim \frac{Z(y)}{f_{1}(y)}, \quad \text { with } \quad \frac{1}{f_{1}(y)}=\sum_{i=0}^{N_{1}} \frac{(-1)^{i} \cdot 1 \cdot 3 \cdots(2 i-1)}{y^{2 i+1}}
$$

where $y \neq 0$ and $Z(y) \neq 0$. Then

$$
\frac{1}{2 \bar{\Phi}(y)} \simeq \frac{1}{2 Z(y)} \cdot f_{1}(y) .
$$

Next, we expend the rational function $f_{1}(y)$ as $\sum_{i=0}^{N_{2}} c_{i} y^{1-2 i}$, a sum of negative powers in $y$, where $N_{2}$ is the order of the expansion to be fixed according to a desired accuracy. To be clear, we list below two examples of $f_{1}(y)\left(N_{1}=2\right.$ or 3$)$ and the associated expansions using $N_{2}=3$ or 5 . 


\begin{tabular}{|c|c|c|c|}
\hline & $f_{1}(y)$ & $N_{2}=3$ & $N_{2}=5$ \\
\hline$N_{1}=2$ & $\left(y^{-1}-y^{-3}+3 y^{-5}\right)^{-1}$ & $y+y^{-1}-2 y^{-3}-5 y^{-5}$ & $y+y^{-1}-2 y^{-3}-5 y^{-5}+y^{-7}+16 y^{-9}$ \\
\hline$N_{1}=3$ & $\left(y^{-1}-y^{-3}+3 y^{-5}-15 y^{-7}\right)^{-1}$ & $y+y^{-1}-2 y^{-3}+10 y^{-5}$ & $y+y^{-1}-2 y^{-3}+10 y^{-5}+31 y^{-7}-29 y^{-9}$ \\
\hline
\end{tabular}

Finally, we obtain the target approximation as follows

$$
\frac{1}{2 \bar{\Phi}(y)} \simeq \frac{1}{2 Z(y)}\left(\sum_{i=0}^{N_{2}} c_{i} y^{1-2 i}\right)
$$

and its accuracy will be determined by an appropriate choice of $N_{1} \geqslant 0$ and $N_{2} \geqslant 1$.

\subsection{Approximation of $l(z, y)$}

The remainder $l(z, y)$ is the actual difference between $\frac{1}{\bar{\Phi}(y+z)+\bar{\Phi}(y-z)}$ and $\frac{1}{2 \bar{\Phi}(y)}$. First, we estimate $\bar{\Phi}(y+z)$ and $\bar{\Phi}(y-z)$ by rational functions in $x$. According to (3.4),

$$
\begin{aligned}
\bar{\Phi}(y+z) & \simeq Z(y+z) \sum_{i=0}^{N_{1}} \frac{(-1)^{i} 1 \cdot 3 \ldots(2 i-1)}{(y+z)^{2 i+1}} \\
& =Z(y) \exp \left(-\frac{2 y z+z^{2}}{2}\right) \sum_{i=0}^{N_{1}} \frac{(-1)^{i} 1 \cdot 3 \ldots(2 i-1)}{(y+z)^{2 i+1}} .
\end{aligned}
$$

Next, we expand the term $\exp \left(-\frac{2 y z+z^{2}}{2}\right) \sum_{i=0}^{N_{1}} \frac{(-1)^{i} 1 \cdot 3 \ldots(2 i-1)}{(y+z)^{2 i+1}}$ in a power series of $z$ according to $z$ 's property $\left(z\right.$ is small) by keeping the four first powers as $e_{0}(y)+e_{1}(y) z+e_{2}(y) z^{2}+$ $e_{3}(y) z^{3}$. More powers in $z$ may be used but we find that the approximation is accurate enough with four terms. Then $\bar{\Phi}(y+z)$ can be written as

$$
\bar{\Phi}(y+z)=Z(y)\left(e_{0}(y)+e_{1}(y) z+e_{2}(y) z^{2}+e_{3}(y) z^{3}\right)+o\left(z^{3}\right)
$$

where each $e_{i}(y)(i=0,1,2,3)$ is a power function in $y$.

Similarly, $\bar{\Phi}(y-z)$ can be written as

$$
\bar{\Phi}(y-z)=Z(y)\left(e_{0}(y)-e_{1}(y) z+e_{2}(y) z^{2}-e_{3}(y) z^{3}\right)+o\left(z^{3}\right) .
$$

According to (3.9) and (3.10) and by assuming $z=0$, we find $e_{0}(y)=\frac{\bar{\Phi}(y)}{Z(y)} \simeq \frac{Z(y)}{f_{1}(y)} \cdot \frac{1}{Z(y)}=$ 
$\frac{1}{f_{1}(y)}$. Thus with the notation $f_{2}(y)=\left(f_{1}(y)\right)^{2} e_{1}(y), l(z, y)$ can be estimated as:

$$
\begin{aligned}
l(z, y) & =\frac{1}{2 \bar{\Phi}(y)}-\frac{1}{\bar{\Phi}(y+z)+\bar{\Phi}(y-z)} \\
& \approx \frac{f_{1}(y)}{2 Z(y)}\left(1-\frac{1}{1+f_{1}(y) e_{2}(y) z^{2}+o\left(z^{3}\right)}\right) \\
& =\frac{f_{1}(y)}{2 Z(y)} \sum_{i=1}^{\infty}(-1)^{i+1}\left(f_{1}(y) e_{2}(y) z^{2}+o\left(z^{3}\right)\right)^{i} \\
& =\frac{z^{2}}{2 Z(y)} \cdot f_{2}(y)+o\left(z^{3}\right) .
\end{aligned}
$$

\begin{tabular}{|c|c|c|}
\hline \multicolumn{2}{|r|}{$N_{1}=2$} & $N_{1}=3$ \\
\hline$e_{2}(y)$ & $\left(\frac{1}{2} y+\frac{15}{2} y^{-5}+45 y^{-7}\right)$ & $\left(\frac{1}{2} y-\frac{105}{2} y^{-7}-420 y^{-9}\right)$ \\
\hline$f_{2}(y)$ & $\left(\frac{1}{2} y+\frac{15}{2} y^{-5}+45 y^{-7}\right)\left(y^{-1}-y^{-3}+3 y^{-5}\right)^{-2}$ & $\left(\frac{1}{2} y-\frac{105}{2} y^{-7}-420 y^{-9}\right)\left(y^{-1}-y^{-3}+3 y^{-5}-15 y^{-7}\right)^{-2}$ \\
\hline$N_{2}=3$ & $\frac{1}{2} y^{3}+y-\frac{3}{2} y^{-1}+\frac{1}{2} y^{-3}+58 y^{-5}$ & $\frac{1}{2} y^{3}+y-\frac{3}{2} y^{-1}+8 y^{-3}-\frac{19}{2} y^{-5}$ \\
\hline$N_{2}=5$ & $\frac{1}{2} y^{3}+y-\frac{3}{2} y^{-1}+\frac{1}{2} y^{-3}+58 y^{-5}+\frac{189}{2} y^{-7}-\frac{401}{2} y^{-9}$ & $\frac{1}{2} y^{3}+y-\frac{3}{2} y^{-1}+8 y^{-3}-\frac{19}{2} y^{-5}-543 y^{-7}-\frac{1391}{2} y^{-9}$ \\
\hline
\end{tabular}

Furthermore, by a combination of the expansions $e_{2}(y)$ and $f_{1}(y)$, we get $f_{2}(y)$ of form $\sum_{i=0}^{N_{2}} d_{i} y^{(3-2 i)}$. We list several examples of $f_{2}(y)$ and their expansions with different values of $N_{1}$ and $N_{2}\left(N_{1}=2\right.$ or $3, N_{2}=3$ or 5$)$.

The final approximation for $l(z, y)$ is

$$
l(z, y) \approx \frac{z^{2}}{2 Z(y)}\left(\sum_{i=0}^{N_{2}} d_{i} y^{(3-2 i)}\right)
$$

By combining (3.8) and (3.12) together with (3.5), finally, we get the following approximation

$$
G\left(a_{0}, b_{0}, h\right) \simeq \frac{1}{2 Z(y)}\left(\sum_{i=0}^{N_{2}} c_{i} y^{1-2 i}\right)-\frac{z^{2}}{2 Z(y)}\left(\sum_{i=0}^{N_{2}} d_{i} y^{(3-2 i)}\right),
$$

where $y=\sqrt{\frac{\mathbf{W}_{0}^{*} h}{m(n-1)}}, z=\frac{\mathbf{Z}_{0}^{*}}{\sqrt{m}}$ and $\mathbf{Z}_{0}^{*} \sim \mathrm{N}(\mathbf{0}, 1) \mathbf{W}_{0}^{*} \sim \chi_{m(n-1)}^{2}$.

Proposition 2. Based on the approximation (3.13), an analytic approximation for $A R L_{0}$ formula is given by

$$
\widehat{A R L_{0}}(h)=\frac{\sqrt{2 \pi}}{2} \sum_{i=0}^{N_{2}}\left(c_{i}-\frac{d_{i}}{m}\right) g_{i}(h)
$$


where

$$
g_{i}(h)=\frac{\Gamma\left(\frac{m n-m}{2}-\frac{2 i-3}{2}\right)\left(1-\frac{h}{m n-m}\right)^{\left(\frac{2 i-3}{2}-\frac{m n-m}{2}\right)}}{2^{\frac{2 i-3}{2}} \Gamma\left(\frac{m n-m}{2}\right)\left(\frac{h}{m n-m}\right)^{\frac{2 i-3}{2}}} .
$$

Proof. By (3.13), (3.1) and $\mathrm{E}\left(z^{2}\right)=\mathrm{E}\left[\frac{\left(\mathbf{z}_{0}^{*}\right)^{2}}{m}\right]=\frac{1}{m}$, we have

$$
\begin{aligned}
\widehat{A R L_{0}}(h) & =\mathrm{E}\left[\frac{1}{2 Z(y)}\left(\sum_{i=0}^{N_{2}} c_{i} y^{(3-2 i)}\right)-\frac{z^{2}}{2 Z(y)}\left(\sum_{i=0}^{N_{2}} d_{i} y^{(3-2 i)}\right)\right] \\
& =\frac{\sqrt{2 \pi}}{2} \sum_{i=0}^{N_{2}}\left(c_{i}-\frac{d_{i}}{m}\right) \mathrm{E}\left[\exp \left(\frac{y^{2}}{2}\right) y^{(3-2 i)}\right] .
\end{aligned}
$$

Let $g_{i}(h)=\mathrm{E}\left[\exp \left(\frac{y^{2}}{2}\right) y^{(3-2 i)}\right]$. Since $\mathrm{W}_{0}^{*} \sim \chi_{m(n-1)}^{2}$ and $y=b_{0} \sqrt{h}=\sqrt{\frac{\mathbf{W}_{0}^{*} h}{m(n-1)}}$ is bounded with high probability, for each $i\left(0 \leqslant i \leqslant N_{2}\right)$, we have

$$
\begin{aligned}
g_{i}(h) & =\mathrm{E}\left[\sum_{k=0}^{\infty} \frac{1}{k !}\left(\frac{y^{2}}{2}\right)^{k} y^{(3-2 i)}\right] \\
& =\sum_{k=0}^{\infty} \frac{1}{2^{k} k !} \mathrm{E}\left[y^{(3-2 i+2 k)}\right] \\
& =\sum_{k=0}^{\infty} \frac{1}{2^{k} k !}\left(\frac{h}{m n-m}\right)^{\frac{3-2 i+2 k}{2}} \mathrm{E}\left[\mathrm{W}_{0}^{* \frac{(3-2 i+2 k)}{2}}\right] \\
& =\sum_{k=0}^{\infty} \frac{1}{2^{k} k !}\left(\frac{h}{m n-m}\right)^{\frac{3-2 i+2 k}{2}} \cdot 2^{\frac{(3-2 i+2 k)}{2}} \frac{\Gamma\left(k+\frac{3}{2}-i+\frac{m n-m}{2}\right)}{\Gamma\left(\frac{m n-m}{2}\right)} \\
& =\frac{\sum_{k=0}^{\infty} \frac{1}{k !}\left(\frac{h}{m n-m}\right)^{k} \Gamma\left(k+\frac{m n-m}{2}-\frac{2 i-3}{2}\right)}{2^{\frac{2 i-3}{2}} \Gamma\left(\frac{m n-m}{2}\right)\left(\frac{h}{m n-m}\right)^{\frac{2 i-3}{2}} .}
\end{aligned}
$$

Here the condition $N_{2}<\frac{m(n-1)+3}{2}$ must be satisfied for the definition of the Gamma function. 
Now, we calculate the numerator of (3.15) with the notation $a=\frac{h}{m n-m}, b=\frac{m n-m}{2}-\frac{2 i-3}{2}$.

$$
\begin{aligned}
\sum_{k=0}^{\infty} \frac{a^{k}}{k !} \Gamma(k+b) & =\sum_{k=0}^{\infty} \frac{a^{k}}{k !} \int_{0}^{\infty} \exp (-t) t^{k+b-1} d t \\
& =\int_{0}^{\infty}\left[\sum_{k=0}^{\infty} \frac{(a t)^{k}}{k !}\right] \exp (-t) t^{b-1} d t \\
& =\int_{0}^{\infty} \exp [-(1-a) t] t^{b-1} d t \\
& =\Gamma(b)(1-a)^{-b},
\end{aligned}
$$

i.e. $\sum_{k=0}^{\infty} \frac{1}{k !}\left(\frac{h}{m n-m}\right)^{k} \Gamma\left(k+\frac{m n-m}{2}-\frac{2 i-3}{2}\right)=\Gamma\left(\frac{m n-m}{2}-\frac{2 i-3}{2}\right)\left(1-\frac{h}{m n-m}\right)^{\left(\frac{2 i-3}{2}-\frac{m n-m}{2}\right)}$.

Substituting (3.16) into (3.15), we get

$$
g_{i}(h)=\frac{\Gamma\left(\frac{m n-m}{2}-\frac{2 i-3}{2}\right)\left(1-\frac{h}{m n-m}\right)^{\left(\frac{2 i-3}{2}-\frac{m n-m}{2}\right)}}{2^{\frac{2 i-3}{2}} \Gamma\left(\frac{m n-m}{2}\right)\left(\frac{h}{m n-m}\right)^{\frac{2 i-3}{2}}},
$$

and finally we have $\widehat{A R L_{0}}(h)=\frac{\sqrt{2 \pi}}{2} \sum_{i=0}^{N_{2}}\left(c_{i}-\frac{d_{i}}{m}\right) g_{i}(h)$.

\section{The approximation orders $N_{1}$ and $N_{2}$}

Our approximation formula (3.14) depends on two parameters, namely, the orders $N_{1}$ and $N_{2}$ introduced in the expansions. These parameters determine the coefficients $\left\{c_{i}\right\}$ and $\left\{d_{i}\right\}$ and finally the accuracy of the approximation (3.14). Below we give some indications on the choice of these parameters.

First, we decide the value of $N_{1}$. The criterion is to choose $N_{1}$ such that the approximation (3.6) of $\bar{\Phi}(y)$ is accurate enough.

According to Abramowitz and Stegun (1965), the difference between $\bar{\Phi}(y)$ and the approximation (3.6)

$$
\frac{Z(y)}{f_{1}(y)}=Z(y) \sum_{i=0}^{N_{1}} \frac{(-1)^{i} 1 \cdot 3 \ldots(2 i-1)}{y^{2 i+1}}
$$

has the form

$$
R_{N_{1}}(y)=\bar{\Phi}(y)-\frac{Z(y)}{f_{1}(y)}=(-1)^{N_{1}+1} \cdot 1 \cdot 3 \cdots\left(2 N_{1}+1\right) \int_{y}^{\infty} \frac{Z(t)}{t^{2 N_{1}+2}} d t
$$

whose absolute value is a decreasing function of $y(y>0)$ when $N_{1}$ is given. So if we choose an $N_{1}$ to ensure $\left|R_{N_{1}}\left(y_{0}\right)\right|<10^{-3}$ at $y_{0}$, then this also holds for any $y>y_{0}$. Since in our application, $y=\sqrt{\frac{\mathbf{W}_{0}^{*} h}{m(n-1)}}$ where $\mathbf{W}_{0}^{*} \sim \chi_{m(n-1)}^{2}$, we can see that $y$ has the magnitude 
of $\sqrt{h}$, which usually ranges from 2 to 10 (usually the $h$ is ranging from 4 to 100 ). So we compare $\bar{\Phi}(y)$ with the approximation $\frac{Z(y)}{f_{1}(y)}=Z(y) \sum_{i=0}^{N_{1}} \frac{(-1)^{i} 1 \cdot 3 \ldots(2 i-1)}{y^{2 i+1}}$ at the point $y_{0}=2$ in order to find an appropriate $N_{1}$.

Table 1: Comparison between $R_{N_{1}}\left(y_{0}\right)$ and $\frac{Z\left(y_{0}\right)}{f_{1}\left(y_{0}\right)}$ when $y_{0}=2$ (accurate to five decimal places)

\begin{tabular}{ccccccc}
$N_{1}$ & 1 & 2 & 3 & 4 & 5 & 6 \\
\hline$R_{N_{1}}\left(y_{0}\right)$ & 0.00250 & -0.00256 & 0.00377 & -0.00730 & 0.01761 & -0.05090 \\
\hline
\end{tabular}

According to Table 1 , we can see that the absolute values of $R_{N_{1}}\left(y_{0}\right)$ are larger than $10^{-3}$ with alternate signs $\left(y_{0}=2\right)$, which are not negligible. In order to reduce the error $R_{N_{1}}\left(y_{0}\right)$, we decide to average the approximation $\frac{Z(y)}{f_{1}(y)}\left(N_{1}=1,2,3,4\right)$ by taking advantage of the $R_{N_{1}}(y)$ 's signs. Equivalently, we average (3.14) when $N_{1}=1,2,3,4$ and $y=2$. Moreover, the averaged approximation (3.4) will perform better when $y \geqslant 2$, since $R_{N_{1}}(y)$ is smaller for larger $y$. As a result, we choose several $N_{1}=1,2,3,4$ rather than choosing a single $N_{1}$ because of $R_{N_{1}}\left(y_{0}\right)$ 's non-negligible values.

The choice of $N_{2}$ directly affects the deviation of $f_{1}(y)=\left(\sum_{i=0}^{N_{1}} \frac{(-1)^{i} 1 \cdot 3 \ldots(2 i-1)}{y^{2 i+1}}\right)^{-1}$ in (3.7) from its Taylor expansions $\sum_{i=0}^{N_{2}} c_{i} y^{1-2 i}$ and the deviation of $f_{2}(y)=\left(f_{1}(y)\right)^{2} e_{2}(y)$ in (3.11) from its Taylor expansions $\sum_{i=0}^{N_{2}} d_{i} y^{(3-2 i)}$. The values of $f_{1}(y)$ and $f_{2}(y)=$ $\left(f_{1}(y)\right)^{2} e_{2}(y)$ converge to their Taylor expansions when $y \geqslant 2$. Therefore, we should select large $N_{2}$ in order to approximate $f_{1}(y)$ and $f_{2}(y)$ precisely by using their Taylor expansions. Then the final approximation can also be more accurate by sharing the same coefficients $\left\{c_{i}\right\}$ and $\left\{d_{i}\right\}$. We choose the smallest $N_{2}$ ensuring $\left|f_{1}(y)-\sum_{i=0}^{N_{2}} c_{i} y^{1-2 i}\right|<$ $10^{-4}$ and $\left|f_{2}(y)-\sum_{i=0}^{N_{2}} d_{i} y^{(3-2 i)}\right|<10^{-4}$. By numerical computation, we obtain several $N_{2}$ 's corresponding to different values of $y$ for $1 \leqslant N_{1} \leqslant 4$ and they are given in Table 2 . 
Table 2: Minimum values of $N_{2}$ for different values of $y$ when $1 \leqslant N_{1} \leqslant 4$ ensuring the error bound $\left|f_{1}(y)-\sum_{i=0}^{N_{2}} c_{i} y^{1-2 i}\right|<10^{-4}$ (top), and ensuring the error bound $\left|f_{2}(y)-\sum_{i=0}^{N_{2}} d_{i} y^{(3-2 i)}\right|<10^{-4}$ (bottom).

\begin{tabular}{ll|c|cccccc}
\hline \hline & & $y$ & 2 & 2.5 & 3 & 3.5 & 4 & 4.5 \\
\hline$N_{1}=1$ & $f_{1}(y)=\frac{1}{\left(\frac{1}{y}-\frac{1}{y^{3}}\right)}$ & $N_{2}$ & 7 & 5 & 3 & 3 & 3 & 3 \\
\hline$N_{1}=2$ & $f_{1}(y)=\frac{1}{\left(\frac{1}{y}-\frac{1}{y^{3}}+\frac{3}{y^{5}}\right)}$ & $N_{2}$ & 11 & 7 & 5 & 5 & 4 & 3 \\
\hline$N_{1}=3$ & $f_{1}(y)=\frac{1}{\left(\frac{1}{y}-\frac{1}{y^{3}}+\frac{3}{y^{5}}-\frac{15}{y^{7}}\right)}$ & $N_{2}$ & 17 & 10 & 7 & 5 & 4 & 4 \\
\hline$N_{1}=4$ & $f_{1}(y)=\frac{1}{\left(\frac{1}{y}-\frac{1}{y^{3}}+\frac{3}{y^{5}}-\frac{15}{y^{7}}+\frac{105}{y^{9}}\right)}$ & $N_{2}$ & 36 & 13 & 9 & 6 & 5 & 5 \\
\hline \hline
\end{tabular}

\begin{tabular}{ll|c|cccccc}
\hline \hline & & $y$ & 2 & 2.5 & 3 & 3.5 & 4 & 4.5 \\
\hline$N_{1}=1$ & $f_{2}(y)=\frac{\left(\frac{1}{2} y-\frac{3}{2} y^{-3}-6 y^{-5}\right)}{\left(\frac{1}{y}-\frac{1}{y^{3}}\right)}$ & $N_{2}$ & 10 & 7 & 6 & 5 & 5 & 4 \\
\hline$N_{1}=2$ & $f_{2}(y)=\frac{\left(\frac{1}{2} y+\frac{15}{2} y^{-5}+45 y^{-7}\right)}{\left(\frac{1}{y}-\frac{1}{y^{3}}+\frac{3}{y^{5}}\right)^{2}}$ & $N_{2}$ & 13 & 10 & 8 & 6 & 6 & 5 \\
\hline$N_{1}=3$ & $f_{1}(y)=\frac{\left(\frac{1}{2} y-\frac{105}{2} y^{-7}-420 y^{-9}\right)}{\left(\frac{1}{y}-\frac{1}{y^{3}}+\frac{3}{y^{5}}-\frac{15}{y^{7}}\right)^{2}}$ & $N_{2}$ & 28 & 14 & 10 & 8 & 7 & 5 \\
\hline$N_{1}=4$ & $f_{1}(y)=\frac{\left(\frac{1}{2} y-\frac{945}{2} y^{-9}-4725 y^{-11}\right)}{\left(\frac{1}{y}-\frac{1}{y^{3}}+\frac{3}{y^{5}}-\frac{15}{y^{7}}+\frac{105}{y^{9}}\right)^{2}}$ & $N_{2}$ & 65 & 18 & 11 & 9 & 6 & 6 \\
\hline \hline
\end{tabular}


From Table 2, we find that for a given $N_{1}$, the value of $N_{2}$ decreases when $y$ increases to ensure an approximation error less than $10^{-4}$ for both $f_{1}(y)$ and $f_{2}(y)$, that is, the appropriate value of $N_{2}$ for $y=2$ will remain appropriate for all $y \geqslant 2$. According to Table 2 and Table 3, we know that the optimal choice of $N_{2}$ when $y=2$ is $N_{2}=65$ to satisfy all the circumstances $1 \leqslant N_{1} \leqslant 4$.

Summarizing the discussion above, we have made our decision to approximate $A R L_{0}$ by choosing $1 \leqslant N_{1} \leqslant 4, N_{2}=65$ and averaging this four cases as follows:

$$
\widehat{A R L_{0}}(h)=\frac{1}{4} \sum_{i=1}^{4} \widehat{A R L_{0}}\left(h \mid N_{1}=i, N_{2}=65\right)
$$

where $\widehat{A R L_{0}}\left(h \mid N_{1}=i, N_{2}=65\right)(i=1,2,3,4)$ are from (3.14). This is the final approximation used for the remaining of the paper.

\section{Monte-Carlo evaluation of the proposed analytic $A R L$ approximation}

The previous section lays the groundwork that allows us to approximate the values of $A R L_{0}$ of the $T^{2}$ chart with estimated parameters. Here we focus on practical implementation of the estimations.

According to the analytic approximation (4.1), we can obtain a series of control limits with different $m$ and $n$ by setting the right side of the equation (4.1) a given value, for example, $A R L_{0}=200$ or 500 . Then by programming, we can compute the corresponding control limit $h$ and thus the control chart criterion can be confirmed with given $m$ and $n$ when $p=1$.

According to (3.14) and (4.1), we first compute and list each $h\left(N_{1}=i, N_{2}=65\right)$ ensuring $\widehat{A R L_{0}}\left(h \mid N_{1}=i, N_{2}=65\right)=200$ and $500(i=1,2,3,4)$ when $m=50,70,100$ with size of subgroup $n=5,10,15$. The computation is based on Monte Carlo. Then for each group, we average four values of $h\left(N_{1}=i, N_{2}=65\right)(i=1,2,3,4)$ to get final control limit $\bar{h}$ that we propose to use in practice for given $m$ and $n$. The results are given in Tables 3 and 4 for $A R L_{0}$ of value 200 and 500, respectively. In the last column of these tables, the values of the corresponding traditional UCL $h^{*}$ are given for comparison. Recall that given $m, n$ and $A R L_{0}, h^{*}$ is determined in (1.3). These tables show in particular that the actual UCL $h$, hence our approximated value $\bar{h}$, are smaller than the traditional $h^{*}$ as predicted by Theorem 1, Although these differences seem not that big, the corresponding ARL values can be indeed very different due to the large variation of the ARL values.

After computing all the values of control limit $\bar{h}$ for various combination of $m$ and $n$, we next check its accuracy by simulations. The simulation method is based on (1.5) following Champ et al. (2005). For each case with specific $m, n$ and $A R L_{0}$, the number of independent repetitions is 10000 . 
Table 3: Control limits for each $N_{1}=1,2,3,4$ ensuring $A R L_{0}=200$ using $m$ subgroups of size $n$ when $N_{2}=65$ and their averages.

\begin{tabular}{c|c|ccccc|c}
$m$ & $n$ & $h\left(N_{1}=1\right)$ & $h\left(N_{1}=2\right)$ & $h\left(N_{1}=3\right)$ & $h\left(N_{1}=4\right)$ & $\bar{h}$ & $h^{*}$ \\
\hline 50 & 5 & 7.8122 & 7.9149 & 7.8469 & 7.9115 & 7.8714 & 8.2183 \\
& 10 & 7.9035 & 8.0093 & 7.9398 & 8.0052 & 7.9645 & 8.1169 \\
& 15 & 7.9299 & 8.0366 & 7.9665 & 8.0323 & 7.9913 & 8.0882 \\
\hline 70 & 5 & 7.8109 & 7.9121 & 7.8462 & 7.9078 & 7.8693 & 8.1202 \\
& 10 & 7.8761 & 7.9796 & 7.9125 & 7.9748 & 7.9358 & 8.0486 \\
& 15 & 7.8950 & 7.9991 & 7.9316 & 7.9941 & 7.9550 & 8.0283 \\
\hline 100 & 5 & 7.8111 & 7.9113 & 7.8468 & 7.9062 & 7.8689 & 8.0473 \\
& 10 & 7.8569 & 7.9585 & 7.8933 & 7.9532 & 7.9154 & 7.9976 \\
& 15 & 7.8700 & 7.9721 & 7.9066 & 7.9666 & 7.9289 & 7.9835 \\
\hline
\end{tabular}

Table 4: Control limits for each $N_{1}=1,2,3,4$ ensuring $A R L_{0}=500$ using $m$ subgroups of size $n$ when $N_{2}=65$ and their averages.

\begin{tabular}{c|c|ccccc|c}
$m$ & $n$ & $h\left(N_{1}=1\right)$ & $h\left(N_{1}=2\right)$ & $h\left(N_{1}=3\right)$ & $h\left(N_{1}=4\right)$ & $\bar{h}$ & $h^{*}$ \\
\hline 50 & 5 & 9.4637 & 9.5318 & 9.4949 & 9.5238 & 9.5036 & 10.0023 \\
& 10 & 9.5970 & 9.6680 & 9.6296 & 9.6594 & 9.6385 & 9.8557 \\
& 15 & 9.6356 & 9.7074 & 9.6687 & 9.6987 & 9.6776 & 9.8143 \\
\hline 70 & 5 & 9.4719 & 9.5395 & 9.5034 & 9.5312 & 9.5115 & 9.8708 \\
& 10 & 9.5673 & 9.6369 & 9.5998 & 9.6282 & 9.6081 & 9.7675 \\
& 15 & 9.5948 & 9.6650 & 9.6276 & 9.6562 & 9.6359 & 9.7383 \\
\hline \multirow{2}{*}{100} & 5 & 9.4799 & 9.5471 & 9.5115 & 9.5386 & 9.5193 & 9.7734 \\
& 10 & 9.5467 & 9.6154 & 9.5790 & 9.6065 & 9.5869 & 9.7018 \\
& 15 & 9.5660 & 9.6350 & 9.5985 & 9.6261 & 9.6064 & 9.6815 \\
\hline
\end{tabular}


Table 5: Empirical $A R L_{0}$ performance of the approximate control limit $\bar{h}$ with target $A R L_{0}=200$

\begin{tabular}{c|ccc}
$m$ & $n$ & $\bar{h}$ & Empirical ARL (s.e.) \\
\hline \hline 50 & 5 & 7.8714 & $202.2(243.9)$ \\
& 10 & 7.9645 & $200.8(216.7)$ \\
& 15 & 7.9913 & $198.5(213.0)$ \\
\hline 70 & 5 & 7.8693 & $200.4(225.5)$ \\
& 10 & 7.9358 & $199.3(213.9)$ \\
& 15 & 7.9550 & $198.3(209.8)$ \\
\hline \multirow{2}{*}{100} & 5 & 7.8689 & $205.1(220.0)$ \\
& 10 & 7.9154 & $196.0(207.1)$ \\
& 15 & 7.9289 & $200.2(203.6)$ \\
\hline
\end{tabular}

Table 6: Empirical $A R L_{0}$ performance of the approximate control limit $\bar{h}$ with target $A R L_{0}=500$

\begin{tabular}{c|ccc}
$m$ & $n$ & $\bar{h}$ & Empirical $A R L_{0}$ (s.e.) \\
\hline \hline 50 & 5 & 9.5036 & $510.0(691.0)$ \\
& 10 & 9.6385 & $503.8(570.2)$ \\
& 15 & 9.6776 & $510.5(557.1)$ \\
\hline 70 & 5 & 9.5115 & $506.0(612.0)$ \\
& 10 & 9.6081 & $499.4(556.3)$ \\
& 15 & 9.6359 & $504.4(538.7)$ \\
\hline \multirow{2}{*}{100} & 5 & 9.5193 & $505(576)$ \\
& 10 & 9.5869 & $506.4(531.3)$ \\
& 15 & 9.6064 & $505.7(529.4)$ \\
\hline
\end{tabular}

Table 5 and Table 6 give empirical $A R L_{0}$ values for several combinations of $m$ and $n$ when $p=1$ using the control limits $\bar{h}$ for target $A R L_{0}=200$ and 500, respectively. We observe that in both Tables, the empirical values of the $A R L_{0}$ from the approximation UCL $\bar{h}$ are very close to the target one within an error of $2 \%$ at most. One may also observe quite large standard errors of the empirical $A R L_{0}$ which are however common in such Monte-Carlo experiments.

\section{Concluding remarks}

When estimated parameters from a Phase I study are used in the design of a Phase II control chart, it has been widely observed in the literature that the properties of the chart are modified. As a theoretical contribution of the paper, we have provided a rigorous proof of this phenomenon in the paper. Next, in addition to existing Monte-Carlo based 
determination method of control limits, we have proposed an analytic method based on an approximation of the $A R L$ function. However, due to the complexity of the approximation problem, our solution is limited to univariate observations. How to extend such approximation procedure to more general multivariate observations remains an important question to explore in the future.

\section{References}

Abramowitz, M. and Stegun, I. (1965). Handbook of mathematical functions: with formulas, graphs, mathematical tables, volume 55. Dover publications.

Bersimis, S., Psarakis, S. and Panaretos, J. (2006). Multivariate statistical process control charts: an overview. Quality and Reliability Engineering International, 23(5), 517-543.

Champ, C., Jones-Farmer, L. and Rigdon, S. (2005). Properties of the t 2 control chart when parameters are estimated. Technometrics, 47(4), 437-445.

Hotelling, H. (1947). A generalized t test and measure of multivariate dispersion.

Jensen, W., Jones-Farmer, L., Champ, C., Woodall, W., et al. (2006). Effects of parameter estimation on control chart properties: a literature review. Journal of Quality Technology, 38(4), 349-364.

Kim, K., Mahmoud, M. and Woodall, W. (2003). On the monitoring of linear profiles. Journal of Quality Technology, 35(3), 317-328.

Lowry, C. and Montgomery, D. (1995). A review of multivariate control charts. IIE transactions, 27(6), 800-810.

Nedumaran, G. and Pignatiello, J. (1999). On constructing t2 control charts for on-line process monitoring. IIE transactions, 31(6), 529-536.

Ryan, T. (2011). Statistical methods for quality improvement. Wiley.

Sullivan, J. and Woodall, W. (1996). A comparison of multivariate control charts for individual observations. Journal of Quality Technology, 28(4), 398-408.

Wierda, S. (1994). Multivariate statistical process control: recent results and directions for future research. Statistica neerlandica, 48(2), 147-168. 